\title{
Atezolizumab in patients with locally advanced and metastatic urothelial carcinoma who have progressed following treatment with platinum-based chemotherapy: a single arm, phase 2 trial
}

\author{
Jonathan E. Rosenberg, M.D. ${ }^{1}$, Jean Hoffman-Censits, M.D. ${ }^{2}$, Tom Powles, M.D. ${ }^{3}$, Michiel S. \\ van der Heijden, M.D., Ph.D. ${ }^{4}$, Arjun V. Balar, M.D ${ }^{5}$, Andrea Necchi, M.D. ${ }^{6}$, Nancy Dawson, \\ M.D. ${ }^{7}$, Peter H. O'Donnell, M.D. ${ }^{8}$, Ani Balmanoukian, M.D. ${ }^{9}$, Yohann Loriot, M.D., Ph.D ${ }^{10}$, \\ Sandy Srinivas, M.D. ${ }^{11}$, Margitta M. Retz, M.D., Ph.D ${ }^{12}$, Petros Grivas, M.D., Ph.D ${ }^{13}$, Richard \\ W. Joseph, M.D. ${ }^{14}$, Matthew D. Galsky, M.D. ${ }^{15}$, Mark T. Fleming, M.D. ${ }^{16}$, Daniel P. Petrylak, \\ M.D. ${ }^{17}$, Jose Luis Perez-Gracia, M.D., Ph.D ${ }^{18}$, Howard A. Burris, M.D. ${ }^{19}$, Daniel Castellano, \\ M.D. ${ }^{20}$, Christina Canil, M.D. ${ }^{21}$, Joaquim Bellmunt, M.D., Ph.D ${ }^{22}$, Dean Bajorin, M.D. ${ }^{1}$, \\ Dorothee Nickles, Ph.D ${ }^{23}$, Richard Bourgon, Ph.D ${ }^{23}$, Garrett M. Frampton, Ph.D ${ }^{24}$, Na Cui, \\ Ph.D ${ }^{23}$, Sanjeev Mariathasan, Ph.D ${ }^{23}$, Oyewale Abidoye, M.D. ${ }^{23}$, Gregg D. Fine, M.D. ${ }^{23}$, and \\ Robert Dreicer, M.D. ${ }^{25}$
}

${ }^{1}$ Genitourinary Oncology Service, Department of Medicine, Memorial Sloan Kettering Cancer Center, New York, NY, USA ${ }^{2}$ Kimmel Cancer Center Thomas Jefferson University, Philadelphia,

\footnotetext{
Corresponding Author: Jonathan E. Rosenberg, Genitourinary Oncology Service, Department of Medicine, Memorial Sloan Kettering Cancer Center, New York, NY, USA., Phone: 646-422-4461, rosenbj1@ mskcc.org.

Contributors

JER, TP, DPP, DB, NC, OA, GDF, and RD contributed to study design. JER, JH-C, TP, MSvdH, AN, PHO, PG, RWJ, MDG, JLP-G, $\mathrm{HAB}, \mathrm{JB}, \mathrm{DB}, \mathrm{GMF}, \mathrm{SM}$, and RD contributed to data collection. RB and DN contributed to preprocessing and analysis of gene expression data (including assignment of subtype) as well as all statistical analyses related to gene expression, CD8 IHC and mutation load exploratory biomarkers. GMF contributed to analysis method development. SM contributed to data analysis, data interpretation, and writing with regards to biomarkers. OA was the medical monitor for the study and responsible for the database lock, data analysis and interpretation. JER and GDF contributed to editing the manuscript and oversight of author review of the manuscript. JER, GDF, $\mathrm{RB}$, and SM contributed to the design of the manuscript figures. All authors were involved in data analysis and interpretation, the manuscript drafting, review, and approval, and the decision to submit for publication.

Declaration of interests

JER has received non-financial support from Roche Genentech and consulting fees for Agensys, Eli Lilly, Sanofi, and Oncogenex. JH$\mathrm{C}$ has received consulting fees from Roche Genentech. TP has received honoraria from Roche, Bristol Myers Squibb, and Merck and research funding from Roche and AstraZeneca. MSvdH has advisory board agreements with Roche Genentech, Astellas, and AstaZeneca and received grants from Astellas. AVB has received consulting fees from Roche Genentech. AN has received consulting fees from Roche and consulting fees and grants from Merck Sharp \& Dohme. ND has no declarations to disclose. PHO has received honoraria from Genentech, Novartis, Algeta ASA, Bayer and research support from Boehringer Ingelheim. AB has received consulting fees from Bristol Myers Squibb, and Merck. YL has received consulting fees from Roche, Sanofi, Astellas, Janssen, Ipsen, and Bristol Myers Squibb, and received a grant from Sanofi. SS has no declarations to disclose. MMR has no declarations to disclose. PG has consulting agreements with Genentech, Dendreon, Bayer, Myriad Genetics and received grants from Genmab; fees were paid to his institution of Cleveland Clinic Foundation from Merck, Mirati, and Oncogenex. RWJ has consulting and advisory board agreements for BMS, Merck, Nektar, Eisai, Novartis, and Cerulean. MDG has advisory board agreements for Genentech, Merck, Astellas, and Novartis, received consulting fees from BioMotiv, and grants from Novartis, Bristol Myers Squibb, and Celgene. MTF has no declarations to disclose. DPP received grants and consulting fees from Genentech during the conduct of the study, and grants and personal fees from Merck, AstraZeneca, Novartis, Pfizer, and Agensys. JLP-G has received grants from Roche. HAB has no declarations to disclose. DC has no declarations to disclose. CC has speaker and advisory board agreements with Sanofi, Janssen, and Astellas, advisory board agreements with Amgen and Bristol Myers Squibb and received congress travel grants from Sanofi and Novartis. JB has received consulting fees from Genentech. DB has an advisory board agreement with Roche Genentech. GMF is an employee and shareholder of Foundation Medicine. DN, RB, NC, SM, OA, and GDF: are employees and shareholders of Genentech. $\mathrm{RD}$ has received consulting fees from Genentech and Merck.
} 
PA, USA ${ }^{3}$ Barts Cancer Institute ECMC, Barts Health and the Royal Free NHS Trust, Queen Mary University of London, London EC1A 7BE, UK ${ }^{4}$ Department of Medical Oncology, The Netherlands Cancer Institute, Amsterdam, The Netherlands ${ }^{5}$ Genitourinary Cancers Program, Perlmutter Cancer Center, NYU Langone Medical Center, New York, NY, USA ${ }^{6}$ Department of Medical Oncology, Fondazione IRCCS Istituto Nazionale dei Tumori, Milano, Italy ${ }^{7}$ Medstar Georgetown University Hospital, Lombardi Comprehensive Cancer Center, Washington, DC, USA ${ }^{8}$ Section of Hematology/Oncology, Department of Medicine, University of Chicago, Chicago, IL, USA ${ }^{9}$ The Angeles Clinic and Research Institute, Los Angeles, CA, USA ${ }^{10}$ Department of Cancer Medicine, Gustave-Roussy Cancer Campus, Villejuif, University of Paris Sud, France ${ }^{11}$ Division of Oncology/Department of Medicine, Stanford University School of Medicine, Stanford, CA, USA ${ }^{12}$ Department of Urology, Klinikum rechts der Isar, Technische Universität München, Munich, Germany ${ }^{13}$ Department of Hematology and Oncology, Taussig Cancer Institute. Cleveland Clinic, Cleveland, OH, USA ${ }^{14}$ Department of Hematology/Oncology, Mayo Clinic, Jacksonville, FL, USA ${ }^{15}$ Division of Hematology/Oncology, Department of Medicine, Mount Sinai School of Medicine. New York, NY, USA ${ }^{16}$ Virginia Oncology Associates, US Oncology Research. Norfolk, VA, USA ${ }^{17}$ Smilow Cancer Center, Yale University, New Haven, CT, USA ${ }^{18}$ Department of Oncology, Clínica Universidad de Navarra, University of Navarra, Pamplona, Navarre, Spain ${ }^{19}$ Sarah Cannon Research Institute, Nashville, TN, USA and Tennessee Oncology, Nashville, TN, USA ${ }^{20}$ Medical Oncology Department, Genitourinary Oncology Unit, University Hospital 12 de Octubre, Madrid, Spain ${ }^{21}$ Division of Medical Oncology, Department of Medicine, University of Ottawa, The Ottawa Hospital Research Institute, The Ottawa Hospital Cancer Centre, Ottawa, ON, Canada ${ }^{22}$ Lank Center for Genitourinary Oncology, Dana-Farber Cancer Institute, Harvard Medical School, Boston, MA, USA ${ }^{23}$ Genentech, Inc., 1 DNA Way, South San Francisco, CA, USA ${ }^{24}$ Foundation Medicine, Inc. Cambridge, MA, USA ${ }^{25}$ Division Hematology/Oncology, University of Virginia School of Medicine, Charlottesville, VA, USA

\section{Summary}

Background-Patients with metastatic urothelial carcinoma have limited treatment options after failure of platinum-based chemotherapy. This multicenter, single-arm phase 2 trial evaluated atezolizumab, an engineered humanized IgG1 monoclonal antibody that binds selectively to programmed death-ligand 1 (PD-L1), in this population.

Methods-Three hundred and ten patients received atezolizumab (1200 mg, every 3 weeks). PDL1 expression on tumor-infiltrating immune cells (IC) was prospectively assessed by immunohistochemistry. The co-primary endpoints were the objective response rate by RECIST v1.1 and immune modified RECIST. A hierarchical testing procedure was used to test whether the objective response rate was significantly higher than the historical control of $10 \%$ at alpha level of 0.05. Exploratory analyses included assessing the association between The Cancer Genome Atlas (TCGA) molecular subtypes, CD8+ T cell infiltration, mutation load, and clinical outcomes.

Findings-By independent review, objective response rates were 26\% (95\% CI 18 to 36) in the IC $2 / 3$ group, $18 \%$ (95\% CI 13 to 24 ) in the IC $1 / 2 / 3$ group and 15\% (95\% CI 11 to 19) in all patients. With a median follow-up of 11.7 months, ongoing responses were observed in $84 \%$ of responders. The median duration of response was not reached (range $2 \cdot 0^{*}, 13.7 *$ months, 
*censored). The median overall survival was 11.4 months (95\% CI 9.0 to not estimable) in the IC $2 / 3$ group, 8.8 months (95\% CI 7.1 to 10.6 ) in the IC $1 / 2 / 3$, and 7.9 months (95\% CI 6.6 to 9.3 ) in all patients. Grade 3-4 related treatment-related adverse events occurred in $16 \%$ and grade 3-4 immune-mediated adverse events occurred in 5\% of treated patients. Exploratory analyses showed TCGA subtypes and mutation load to be independently predictive for response to atezolizumab.

Interpretation-Atezolizumab demonstrated durable activity and good tolerability in this population. PD-L1 expression on immune cells was associated with response. This is the first report to show the association of TCGA subtypes with response to immune checkpoint inhibition and demonstrate the importance of mutation load as a biomarker of response to this class of agents in advanced urothelial carcinoma.

Funding-F. Hoffmann-La Roche Ltd.

\section{Introduction}

Urothelial carcinoma kills over 165,000 patients annually worldwide and is the ninth most common cancer overall. ${ }^{1,2}$ The efficacy of immunotherapy in non-muscle invasive urothelial carcinoma of the bladder was first established in 1976 with Bacillus Calmette-Guerin, but no immunotherapy has been approved for the treatment of advanced disease. ${ }^{3}$ Platinum-based chemotherapy is the standard of care in treatment-naïve patients with metastatic urothelial carcinoma, and is associated with an overall survival of approximately 9-15 months. ${ }^{4,5}$ The prognosis for patients who relapse after platinum-based therapy is dismal, with median survival ranging from five to seven months and no known life-prolonging treatments. ${ }^{6}$ Novel approaches are needed to break this therapeutic stalemate.

Programmed death ligand 1 (PD-L1) is an immune checkpoint that negatively regulates $\mathrm{T}$ cell function by binding to its receptors programmed death-1 (PD-1) or B7-1 on activated T lymphocytes and other immune cells. Because $\mathrm{T}$ lymphocytes play a central role in mediating acquired antitumoral immunity, expression of PD-L1 in the tumor microenvironment endows tumors with a mechanism to evade eradication by the host immune system..$^{7-9}$ PD-L1 is broadly expressed across a wide range of malignancies, including urothelial carcinoma, and blockade of the PD-L1/PD-1 pathway has been shown to produce overall survival benefits in non-small cell lung cancer, melanoma and renal cell carcinoma. ${ }^{7,10-15}$

Recent data has suggested that immune checkpoint inhibitors are more active in tumors with high mutation rates. ${ }^{11,16-22}$ Emerging data from The Cancer Genome Atlas (TCGA) indicated that urothelial carcinoma carried the third highest mutation rate and that gene expression signatures could be used to separate the disease into luminal and basal subtypes. ${ }^{23,24}$ Additional mechanisms such as increased prevalence of non-synonymous mutations, higher neoantigen load, higher antigen binding affinity, and select $\mathrm{T}$ effector signatures, have all been identified as factors that may predict for durable clinical benefit in patients treated with immune checkpoint inhibitors consistent with the hypothesis that mutations may create neoantigens that are recognized by anti-tumor T cells. ${ }^{25-29}$ Taken together, these observations provided a rationale for the clinical investigation of anti-PD-L1 immunotherapy in metastatic urothelial cancer. 
Atezolizumab is an engineered humanized monoclonal immunoglobulin-G1 antibody that binds selectively to PD-L1 and prevents its interaction with PD-1 and B7.1 while sparing the interaction between PD-L2 and PD-1. ${ }^{30,31}$ Atezolizumab has demonstrated durable responses in a cohort of metastatic bladder cancer patients in a phase 1 study with higher response rates observed in patients with higher levels of PD-L1 expression on tumor infiltrating immune cells. ${ }^{32}$

To confirm the antitumor activity of atezolizumab in patients with advanced urothelial carcinoma whose disease had progressed after prior platinum-based chemotherapy, we conducted a phase 2 , global, multicenter, single-arm trial to evaluate the efficacy and safety of atezolizumab. Prospective evaluation of the association of PD-L1 expression with response was a co-primary endpoint. In addition, exploratory translational studies were conducted to address the scientific hypotheses associated with checkpoint inhibition in metastatic urothelial carcinoma.

\section{Methods}

\section{Study Oversight and Conduct}

The study (ClinicalTrials.gov number, NCT02108652) was designed, conducted, and analyzed by employees of F. Hoffmann-La Roche Ltd, the Sponsor, in collaboration with the study investigators. The study was approved by the independent review board at each participating site and was conducted in full conformance of the provisions of the Declaration of Helsinki and the Good Clinical Practice Guidelines. An independent Data Monitoring Committee reviewed the available safety data every 6 months after the first patient enrolled. The sponsor and the authors conducted data analyses and manuscript writing.

\section{Study Design and Treatment}

This was a phase 2, global, multicenter, single-arm two-cohort trial (figure 1, appendix). Cohort 1 consisted of patients who were treatment-naïve in the metastatic setting and considered to be cisplatin-ineligible and are not described in this report. Cohort 2 which consisted of patients with inoperable locally advanced or metastatic urothelial carcinoma whose disease had progressed after prior platinum-based chemotherapy received a fixed dose of $1200 \mathrm{mg}$ intravenous atezolizumab administered on Day 1 of each 21-day cycle. Dose interruptions were allowed, but dose reductions were not permitted. Patients were informed of the potential for pseudoprogression as part of the consent process, and advised to discuss treatment beyond progression with their study physician. Patients were permitted to continue atezolizumab treatment after RECIST v1.1 criteria for progressive disease if they met pre-specified criteria for clinical benefit to allow for identification of non-conventional responses.

The primary endpoint of this study was objective response rate based upon two distinct methods: independent review facility (IRF)-assessed per RECIST version 1.1, and investigator-assessed per immune modified RECIST criteria to better evaluate atypical response kinetics observed with immunotherapy (see Study Protocol). ${ }^{33,34}$ Co-primary endpoints were chosen due to the emerging recognition that RECIST v1.1 may be 
inadequate to fully capture the benefit of the unique patterns of response from immunotherapeutic agents. ${ }^{35}$

Secondary endpoints included: duration of response and progression-free survival by both IRF per RECIST v1.1 and investigator assessed per immune modified RECIST, overall survival, 12-month overall survival, and safety. Exploratory analyses included the association between gene expression profiling, CD8+ T cell infiltration, and mutation load with IRF-assessed objective response.

\section{Patients}

Patients were eligible for enrollment in the study if they had histologically or cytologically documented locally advanced (T4b, any N; or any T, N 2-3) or metastatic (M1, Stage IV) urothelial carcinoma (including renal pelvis, ureter, urinary bladder, or urethra). Eligible patients had an Eastern Cooperative Oncology Group performance status (ECOG PS) of 0 or 1; measurable disease defined by RECIST v1.1; adequate hematologic and end-organ function; and no autoimmune disease or active infections. Formalin-fixed paraffin-embedded tumor specimens with sufficient viable tumor content were required prior to study enrollment.

\section{Study Assessments}

Measurable and evaluable lesions were assessed and documented prior to treatment. Patients underwent tumor assessments every 9 weeks for the first 12 months following Cycle 1, Day 1. After 12 months, tumor assessments were performed every 12 weeks. Safety assessments were performed according to National Cancer Institute Common Terminology Criteria for Adverse Events (NCI CTCAE), Version 4.0. A sample of archived tumor tissues, as well as serum and plasma samples, was collected for exploratory biomarker assessments.

\section{PD-L1 Immunohistochemistry}

Patient tumor samples were prospectively and centrally assessed for PD-L1 expression by immunohistochemistry using the Ventana SP142 assay. ${ }^{36}$ The PD-L1 tumor-infiltrating immune cell (IC) status was defined by the percentage of PD-L1 positive ICs: IC0 $(<1 \%)$; IC1 ( $\geq 1 \%$ but $<5 \%$ ); and IC $2 / 3$ ( $\geq 5 \%$ ). Areas of BCG inflammatory response were excluded from the assessment of PD-L1 IC status. An analysis of PD-L1 expression on tumor cells and CD8+ infiltration by immunohistochemistry was also performed. ${ }^{30}$ (See the Supplementary Appendix for additional details).

\section{Exploratory Biomarker Analyses}

Gene expression levels were quantified by Illumina TruSeq RNA Access RNA-seq. ${ }^{37-39}$ Molecular subtypes were assigned following TCGA, with some modifications to adapt for the use of RNA Access RNA-seq platform for FFPE tissues from our study. ${ }^{23}$ Mutation detection and mutation load assessment as estimated by targeted genomic profiling were performed by Foundation Medicine. ${ }^{40}$ These analyses were performed on tumor tissue collected during screening (see the appendix for additional details). 


\section{Statistical Analysis}

Efficacy analyses were based on the intent to treat population. Objective response rate was determined on the objective response-evaluable population, defined as intent to treat patients who had measureable disease per RECIST v1.1 at baseline, and duration of response analyses were performed on the subset of patients who achieved an objective response. The exact binomial test was used to test the binary endpoints of objective response rate. The time-to-event outcomes, including duration of response, progression free survival and overall survival were estimated using the Kaplan-Meier method. ${ }^{41}$ The $95 \%$ CIs for median duration of response, progression free survival and overall survival were computed using a robust nonparametric Brookmeyer and Crowley method. ${ }^{42}$

For the primary endpoint of objective response rate, a hierarchical fixed-sequence testing procedure was used to compare the objective response rate between the treatment arm and a historical control for three pre-specified populations in the following order: objective response-evaluable patients with a PD-L1 IHC score of IC2/3, followed by IC1/2/3, followed by all objective response-evaluable patients (table 1 , appendix). The hypothesis tests on these three populations were sequentially performed on the basis of IRF-assessed objective response rate according to RECIST v1.1 followed by the investigator-assessed objective response rate according to immune modified RECIST at a specific two-sided a level of 0.05 for each test, while controlling the overall Type I error at the same a level. If no statistical significance was detected at a particular level in the hierarchy, then no further hypothesis testing occurred. The study was designed to estimate the objective response rate in patients receiving atezolizumab and to detect an improvement in the objective response rate compared to $10 \%$. No formal alternative objective response rate hypothesis was chosen. The study had a variable range of statistical power at different alternative objective response rates. We planned to enroll a minimum of approximately $100 \mathrm{IC} 2 / 3$ patients resulting in an overall sample size of approximately 300 patients based upon an estimated $30 \%$ prevalence for the IC $2 / 3$ population. The $95 \%$ CI using the Clopper-Pearson method for an observed objective response rate of $40 \%$ was $30 \%$ to $50 \%$, and the study would have $100 \%$ power to detect a $30 \%$ increase in objective response rate from $10 \%$ to $40 \% .{ }^{43}$ Alternatively, the $95 \%$ CI using the Clopper-Pearson method for an observed objective response rate of $20 \%$ was $13 \%$ to $29 \%$, and the study would have $85 \%$ power to detect a $10 \%$ increase from $10 \%$ to $20 \%$. The primary analysis was triggered by a minimum of 24-weeks of follow-up from the last patient enrolled. This report used a later data cutoff of September 14, 2015 to explore duration of response.

Safety analyses were performed on all treated patients, defined as enrolled patients who received any amount of the study drug. We performed analyses of objective response rate in pre-specified subgroups based on known baseline prognostic factors and reported descriptively. No formal hypothesis testing was planned. Additional biomarker analyses beyond PD-L1 IC were exploratory only and not pre-specified. The biomarker evaluable population was based upon the objective response-evaluable population who had available associated gene expression and mutational load data. 


\section{Role of the funding source}

The funder of the study was involved in the study design, data collection, data analysis, data interpretation, and writing of the report and gave approval to submit. The corresponding author and co-authors were involved in the study design, data interpretation, had full access to all the data in the study, the writing of the report, and had final responsibility for the decision to submit for publication.

\section{Results}

A total of 486 patients were screened and 315 patients were enrolled on the study in Cohort 2 between May 2014 and November 2014 (figure 1 and figure 1, appendix). Three hundred and ten patients received at least one dose of atezolizumab and were evaluable for efficacy and safety. At the time of the data cutoff on September 14, 2015, 202 patients (65\%) had discontinued treatment (193 patients had died, 8 due to withdrawal by patient and 1 due to other reasons).

Table 1 summarizes the baseline characteristics of the patients. Forty one percent of patients had received two or more prior systemic regimens for metastatic disease. Many patients had adverse prognostic risk factors, including, visceral and/or liver metastasis at study entry (78\% and $31 \%$, respectively), and baseline hemoglobin $<10 \mathrm{~g} / \mathrm{dL}(22 \%)$.

Tissue for PD-L1 immunohistochemistry analysis consisted of surgical resection specimens $(n=215)$, biopsies (i.e., core needle or forceps) from primary lesions $(n=23)$ or metastatic sites $(n=41)$, transurethral resection of bladder tumor (TURBT) samples $(n=29)$ and biopsy from unknown lesion $(n=2)$. PD-L1 IC2/3 prevalence was higher in resection and TURBT specimens versus biopsies from primary lesions or metastatic sites (39\% and 34\% versus $17 \%$ and $8 \%$, respectively). PD-L1 IC2/3 prevalence in primary tumor samples (regardless of specimen type) was 33\%, $(\mathrm{n}=233)$ while $\mathrm{PD}-\mathrm{L} 1 \mathrm{IC} 2 / 3$ prevalence in metastatic tumor samples was $28 \%(\mathrm{n}=78)$. Patients were evenly distributed between the PD-L1 IC groups; IC0 (33\%), IC1 (35\%), and IC2/3 (32\%). Baseline characteristics were well balanced between the IC2/3 group, IC1/2/3 group, and the intent to treat population (table 1 ).

The primary analysis demonstrated that treatment with atezolizumab resulted in a significantly improved RECIST v1.1 objective response rate for each pre-specified IC group [IC2/3, 27\% (95\% CI 19 to 37), p<0.0001; IC1/2/3, 18\% (95\% CI 13 to 24), $\mathrm{p}=0.0004$; and all patients, $15 \%$ ( $95 \% \mathrm{CI}, 11$ to 20 ), $\mathrm{p}=0.0058$ ] compared to a historical control overall response rate of $10 \%$ (table 2, appendix). The updated analysis of efficacy described herein was later conducted to assess the durability of response (table 2). By independent radiological review (RECIST v1.1), the updated analysis of efficacy showed an objective response rate of $26 \%$ (95\% CI 18 to 36 ) in the IC2/3 group, including $11 \%$ of patients who achieved a complete response. In the IC1/2/3 group, the objective response rate was $18 \%$ (95\% CI 13 to 24), with complete response observed in 13 patients (6\%). For all evaluable patients, the objective response rate was 15\% (95\% CI 11 to 19); with complete response observed in 15 patients (5\%) (figure 2, appendix). Investigator-assessed response rates (per immune modified RECIST) were similar to the RECIST v1.1 results (table 2). With a 
median follow-up of 11.7 months, the median duration of response was not yet reached in any of the PD-L1 immunohistochemistry groups (range, 2.0*, 13.7* months, *censored values) (data for IC $2 / 3$ group is shown in figure $2 \mathrm{~A}-\mathrm{C}$; IC0 and IC1 groups shown in figure 3 , appendix). At the time of the data cut-off, ongoing responses were observed in 38 of the 45 responding patients (84\%). The median time to response was 2.1 months (95\% CI 2.0 to $2 \cdot 2)$.

To account for the occurrence of pseudoprogression, patients were allowed to continue treatment beyond IRF RECIST v1.1 progression. One hundred and twenty one patients were treated beyond progression for a median of 7.8 weeks, and of these, 21 (17\%) subsequently experienced target lesion reduction of at least $30 \%$ from their baseline scans (figure 4 , appendix).

Durable responses observed included patients with upper tract disease and patients with poor prognostic features. While the presence of liver metastasis in patients resulted in a lower objective response rate compared to patients with no liver metastases (5\% vs $19 \%$, table 3 , appendix), these responses were durable with the median duration of response not reached at the time of the data cut-off (95\% CI not estimable). A similar trend was observed in patients with visceral metastases (10\% vs 31\% for patients with no visceral metastases) and ECOG PS 1 (8\% vs 25\% for patients with ECOG PS 0). The absence of visceral metastasis (i.e., lymph-node only disease) at baseline was associated with the highest complete response rate. The median duration of response was not yet reached across any subgroup analyzed.

With a median survival follow-up of approximately 11.7 months (range, $0.2 *$ to $15 \cdot 2$; *denotes a censored value) the median progression-free survival (RECIST v1.1) was 2.1 months among all patients (95\% CI $2 \cdot 1$ to $2 \cdot 1$ ) and similar across all IC groups. The investigator-assessed median progression-free survival by immune modified RECIST criteria was 4.0 months ( $95 \%$ CI 2.6 to 5.9 ) in the IC $2 / 3$ group compared to 2.9 months (95\% CI 2.1 to 4.1 ) in the IC1/2/3 group and 2.7 months ( $95 \%$ CI 2.1 to 3.9 ) in all patients.

The median overall survival was 11.4 months ( $95 \%$ CI 9.0 to not estimable) for the IC $2 / 3$ group, 8.8 months ( $95 \%$ CI $7 \cdot 1$ to 10.6 ) in the IC1/2/3 group, and 7.9 months (95\% CI 6.6 to 9.3) for the entire cohort of patients, (figure 2D). The 12-month landmark overall survival rate was $48 \%$ in the IC $2 / 3$ (95\% CI 38 to 58) group, $39 \%$ in the IC $1 / 2 / 3$ (95\% CI 32 to 46 ) group and $36 \%$ (95\% CI 30 to 41 ) in the intent to treat population. In patients who received only one prior line of therapy $(\mathrm{n}=124)$ in the metastatic setting and no prior adjuvant/ neoadjuvant therapy, the median overall survival was not estimable (95\% CI 9.3 to not estimable) for the IC $2 / 3$ group, 10.3 months (95\% CI 7.5 to 12.7 ) in the IC $1 / 2 / 3$ group, and 9.0 months (95\% CI $7 \cdot 1$ to 10.9$)$ for the entire second-line population.

The median duration of treatment was 12 weeks (range, 0 to 66). All cause, any grade adverse events were reported in $97 \%$ of patients, with $55 \%$ of patients experiencing a grade 3-4 event (table 4, appendix). Sixty-nine percent of patients had a treatment-related adverse event of any grade, and $16 \%$ of patients had a grade 3-4 related adverse event. Treatmentrelated serious adverse events were observed in $11 \%$ of patients. There were no treatmentrelated deaths reported on study. The majority of treatment-related adverse events were mild 
to moderate in nature, with fatigue (30\%), nausea (14\%), decreased appetite (12\%) pruritus $(10 \%)$, pyrexia (9\%), diarrhea (8\%), rash (7\%) and arthralgia (7\%) among the most common any grade events (table 3 ). The incidence of grade 3-4 treatment-related adverse events was low, with fatigue the most commonly occurring at $2 \%$ (table 3 ). There were no reports of febrile neutropenia.

Seven percent of patients had an immune-mediated adverse event of any grade, with pneumonitis $(2 \%)$, increased aspartate aminotransferase (1\%), increased alanine aminotransferase (1\%), rash (1\%) and dyspnea (1\%) being the most common adverse events. Five percent had a grade 3-4 immune-mediated adverse event (all cause). No immunemediated renal toxicity was observed. Thirty percent of patients had an adverse event leading to dose interruption. Four percent of patients experienced an adverse event that lead to treatment withdrawal. Twenty-two percent (69/310) of patients had an adverse event requiring systemic steroid use.

Exploratory translational analyses showed that PD-L1 immunohistochemistry expression on tumor infiltrating immune cells (IC) was associated with expression of genes in a CD8 T effector set $\left(\mathrm{T}_{\text {eff }}\right)$ (figure 3A; figure 5A, appendix). Among genes in the $\mathrm{T}_{\text {eff }}$ set, responses to atezolizumab were most closely associated with high expression of two interferon- $\gamma$ inducible Thelper $1\left(\mathrm{~T}_{\mathrm{H}} 1\right)$-type chemokines, CXCL9 $(\mathrm{p}=0.0057)$ and CXCL10 $(\mathrm{p}=0.0079$, figure 3B). A similar, though less pronounced, trend was also seen with respect to other genes in the set (figure 5B, appendix). Consistent with increased T-cell trafficking chemokine expression, tumor center CD8+ T cell infiltration was also associated with both PD-L1 IC ( $p<0.0001$, figure 3C) and response to atezolizumab ( $p=0.0265$, figure 3D).

After adapting the TCGA classification approach for use with our expression assay, gene expression analysis $(\mathrm{n}=195)$ was used to classify patients into luminal $(\mathrm{n}=73)$ and basal $(\mathrm{n}=122$ ) subtypes as defined by TCGA (figure 6, appendix). PD-L1 IC prevalence was highly enriched in the basal subtype versus the luminal subtype (60\% vs $23 \%, \mathrm{p}<0.0001$, figure 3E) with IC2/3 expression of $15 \%$ in the papillary-like luminal cluster I, $34 \%$ in the cluster II, 68\% in the squamous-like basal cluster III and 50\% in the basal cluster IV subtype. Elevated PD-L1 tumor cell expression was almost exclusively seen in the basal subtype (39\% in basal vs $4 \%$ in luminal, $\mathrm{p}<0 \cdot 0001$, figure $3 \mathrm{~F}$ ) and did not correlate with objective response rate. Consistent with PD-L1 IC2/3 expression, CD8 T-effector gene expression was elevated in luminal cluster II and basal cluster III/IV and not in luminal cluster I (figure 6, appendix). Response to atezolizumab occurred in all TCGA subtypes but was significantly higher in the luminal cluster II subtype than in other subtypes, which demonstrated an objective response rate of $34 \%$ ( $\mathrm{p}=0.0017$, figure $3 \mathrm{G}$ ).

Mutation load was estimated in 150 patients by examining a representative panel of 315 cancer-related genes. The median mutation load was significantly increased in responders $(12 \cdot 4 / \mathrm{Mb})$ compared to non-responders $(6.4 / \mathrm{Mb})(\mathrm{p}<0.0001$, figure $3 \mathrm{H})$. The relationship between mutation load and response was unrelated to TCGA subtype $(\mathrm{p}=0 \cdot 2200$, figure $3 \mathrm{H}$ ) or IC subgroup (figure 7, appendix). A subgroup analysis of only those patients with bladder primary tumors, (figure 8, appendix), produced essentially equivalent results. Finally, 
smoking status did not correlate with mutation load $(\mathrm{p}=0.2454)$ or with response $(\mathrm{p}=0.5373)$ to atezolizumab.

\section{Discussion}

Since the development of combination treatment with methotrexate, vinblastine, doxorubicin and cisplatin chemotherapy thirty years ago, there have been no major improvements in the treatment outcomes for patients with urothelial carcinoma. ${ }^{44}$ The results of this large single arm phase 2 study show that atezolizumab induced durable anti-tumor responses in patients with advanced urothelial carcinoma whose tumors have progressed during or after platinumbased chemotherapy. This trial included heavily pre-treated patients and notably, the median duration of response had not been reached despite a median follow-up of 11.7 months. The low incidence of clinically relevant treatment-related adverse events makes atezolizumab widely applicable in this patient population who often has multiple co-morbidities and/or renal impairment. This durable efficacy and tolerability is striking in comparison to outcomes observed with currently available second-line chemotherapy for urothelial carcinoma. ${ }^{6,45,46}$

The 12-month overall survival rate in the entire cohort that included approximately $41 \%$ of patients treated in the third- or later-line was $48 \%$ (95\% CI 38 to 58) in the IC2/3 group, $39 \%$ in the IC $1 / 2 / 3$ (95\% CI 32 to 46 ) group and $36 \%$ (95\% CI 30 to 41 ) in the ITT population. These overall survival results compare favorably to a landmark 12-month survival rate of $20 \%$ (95\% CI 17 to 24) from a pooled analysis of ten phase 2 trials that evaluated 646 patients who received second-line chemotherapy or biologics. ${ }^{47}$

Currently, the prognostic value of PD-L1 IC expression is unknown, with conflicting reports in the literature, although it does not appear to be associated with validated adverse risk factors in this data set. ${ }^{48,49}$ Therefore, it appears likely that the improved survival in this patient population is related to atezolizumab treatment. The results of ongoing randomized studies (NCT02302807) are needed to appropriately assess the prognostic and predictive value of the Ventana SP142 immunohistochemistry assay, and to better understand which patients derive clinical benefit.

Responses to atezolizumab were associated with both conventional RECIST as well as atypical response kinetics, with an additional $17 \%$ of patients treated beyond progression having shrinkage (at least 30\% reduction) of target lesions following RECIST v1.1 progression. The median progression-free survival was similar across the immunohistochemistry subsets with RECIST v1.1; however, it increased when immune modified RECIST criteria were utilized to account for the non-classical responses that may be observed with cancer immunotherapy. In this study, a disconnect between progressionfree survival and overall survival was observed, similar to other immune checkpoint agents in other diseases, further suggesting that modifications of RECIST v1.1 are needed to better capture the benefit of immunotherapy treatment.

This study required a tumor specimen to be submitted during screening for prospective PDL1 testing using the Ventana SP142 assay. In a pre-specified analysis, higher levels of PD- 
L1 immunohistochemistry expression on immune cells were associated with a higher response rate to atezolizumab and longer overall survival. In contrast, the frequency of PDL1 expression on tumor cells was low and did not show an association with objective response, lending further support to the importance of adaptive immunity in driving clinical benefit to immune checkpoint inhibitors.

Similarly, the association of immune activation gene subsets (e.g., CXCL9, and CD8A) and other immune checkpoint genes (PD-L1, CTLA-4, TIGIT, data not shown) with IC PD-L1 expression suggests that the IC PD-L1 expression represents adaptive immune regulation and the presence of a pre-existing (but suppressed) immune response in urothelial carcinoma tumors. ${ }^{30}$ The presence of other negative regulators (e.g., TIGIT) further suggests that combination immunotherapeutic approaches may further enhance responses.

In addition to PD-L1 immunohistochemistry expression on immune cells, response to atezolizumab was strongly correlated with mutation load. This association was independent of the association between TCGA subtype or PD-L1 IC score and response (figure 9, appendix). This study employed a novel approach to interrogating the FoundationOne panel that covers $\sim 3 \%$ of the exome to estimate mutation load. Although this targeted approach interrogated a much smaller fraction of the exome than typically used for mutation load estimation, a re-analysis of TCGA bladder urothelial carcinoma mutation data showed that whole-exome results were well-correlated with those obtained from only the FoundationOne regions (figure 10, appendix). Moreover, the correlation of mutational load and response to atezolizumab is consistent with the pattern observed in other malignancies, and reinforces the concept that the multiple mutations that occur in cancer create novel epitopes against which protective $\mathrm{T}$ cell responses are directed. ${ }^{25}$

Interestingly, the molecular subtypes identified by the TCGA analysis were also associated with response to atezolizumab, suggesting that in addition to PD-L1 expression, subtypes differed in underlying immune biology. While responses were observed across all TCGA subtypes, significantly higher response rates were observed in the luminal cluster II subtype, which was characterized by transcriptional signatures associated with the presence of activated T effector cells. In contrast, luminal cluster I was associated with low expression of CD8+ effector genes, lower PD-L1 IC/TC expression and lower responses to atezolizumab, consistent with a landscape often devoid of pre-existing immune activity. Basal clusters III and IV were also associated with increased PD-L1 IC expression as well as CD8+ effector genes. However, unlike luminal cluster II, basal clusters III/IV also exhibited high PD-L1 $\mathrm{TC}$ expression. The reduced response rates in the basal subtypes compared to luminal cluster II strongly suggests that other immunosuppressive factors exist in the basal subtypes that prevent effective $\mathrm{T}$ cell activation with inhibition of the PD-L1/PD-1 pathway. The differences in the immune landscape of luminal versus basal subtypes highlight the need to further understand the underlying immune biology to develop future rational combination or sequential treatment strategies.

While PD-L1 IC status clearly is associated with atezolizumab response, incorporation of TCGA gene expression subtype, mutation load, or both of these novel biomarkers into a model based on PD-L1 IC staining significantly improved the association with response 
(figure 9, appendix). Thus, disease subtype and mutation load do not simply recapitulate the information already provided by PD-L1 expression in immune cells, but rather, they provide independent and complementary information. Additional data and larger sample sizes are required to allow the formal construction of a multi-marker classifier, and continued consideration of all three biomarkers is warranted in next generation companion diagnostics.

In conclusion, we report that targeting PD-L1 with atezolizumab is effective in heavily pretreated locally advanced or metastatic urothelial carcinoma patients, and that responses are more common in patients with higher levels of PD-L1 expression on immune cells. The efficacy appears to be driven by underlying genomic, molecular and immunologic factors.

\section{Supplementary Material}

Refer to Web version on PubMed Central for supplementary material.

\section{Acknowledgments}

We thank the patients and their families who participated in the study. We would also like to thank all of the investigators and their staff. We thank Mark Kockx at Histogenix for technical assistance; Fatema LeGrand, Xiaodong Shen, and AnnChristine Thåström at Genentech for contributions to the study; Shi Li at Genentech for contributions to the primary data analysis; Priti Hegde at Genentech for input on biomarker strategy and the manuscript; Zach Boyd at Genentech for contributions to the biomarker analysis; and Cathleen Ahearn and Daniel Chen at Genentech for input into the study design and the manuscript. Medical writing assistance for this manuscript was provided by Peter Flanagan associated with Eolas Communications, and paid for by F. HoffmannLa Roche Ltd.

\section{References}

1. Ferlay J, Soerjomataram I, Dikshit R, et al. Cancer incidence and mortality worldwide: sources, methods and major patterns in GLOBOCAN 2012. Int J Cancer. 2015; 136:E359-86. [PubMed: 25220842]

2. Torre LA, Bray F, Siegel RL, et al. Global cancer statistics, 2012. CA Cancer J Clin. 2015; 65:87108. [PubMed: 25651787]

3. Morales A, Eidinger D, Bruce AW. Intracavitary Bacillus Calmette-Guerin in the treatment of superficial bladder tumors. J Urol. 1976; 116:180-3. [PubMed: 820877]

4. von der Maase H, Sengelov L, Roberts JT, et al. Long-term survival results of a randomized trial comparing gemcitabine plus cisplatin, with methotrexate, vinblastine, doxorubicin, plus cisplatin in patients with bladder cancer. J Clin Oncol. 2005; 23:4602-8. [PubMed: 16034041]

5. De Santis M, Bellmunt J, Mead G, et al. Randomized phase II/III trial assessing gemcitabine/ carboplatin and methotrexate/carboplatin/vinblastine in patients with advanced urothelial cancer who are unfit for cisplatin-based chemotherapy: EORTC study 30986. J Clin Oncol. 2012; 30:1919. [PubMed: 22162575]

6. Bellmunt J, Théodore C, Demkov T, et al. Phase III trial of vinflunine plus best supportive care compared with best supportive care alone after a platinum-containing regimen in patients with advanced transitional cell carcinoma of the urothelial tract. J Clin Oncol. 2009; 27:4454-61. [PubMed: 19687335]

7. Dong H, Strome SE, Salomao DR, et al. Tumor-associated B7-H1 promotes T-cell apoptosis: a potential mechanism of immune evasion. Nat Med. 2002; 8:793-800. [PubMed: 12091876]

8. Marincola FM, Jaffee EM, Hicklin DJ, Ferrone S. Escape of human solid tumors from T-cell recognition: Molecular mechanisms and functional significance. Adv Immunol. 2000; 74:181-273. [PubMed: 10605607] 
9. Iwai Y, Ishida M, Tanaka Y, et al. Involvement of PD-L1 on tumor cells in the escape from host immune system and tumor immunotherapy by PD-L1 blockade. Proc Natl Acad Sci USA. 2002; 99:12293-7. [PubMed: 12218188]

10. Inman BA, Sebo TJ, Frigola X, et al. PD-L1 (B7-H1) expression by urothelial carcinoma of the bladder and BCG-induced granulomata: associations with localized stage progression. Cancer. 2007; 109:1499-505. [PubMed: 17340590]

11. Garon EB, Rizvi NA, Hui R, et al. Pembrolizumab for the treatment of non-small-cell lung cancer. N Engl J Med. 2015; 372:2018-28. [PubMed: 25891174]

12. Robert C, Long GV, Brady B, et al. Nivolumab in Previously Untreated Melanoma without BRAF Mutation. N Engl J Med. 2015; 372:320-30. [PubMed: 25399552]

13. Motzer RJ, Escudier B, McDermott DF, et al. Nivolumab versus everolimus in advanced renal-cell carcinoma. N Engl J Med. 2015; 373:1803-13. [PubMed: 26406148]

14. Brown JA, Dorfman DM, Ma FR, et al. Blockade of programmed death-1 ligands on dendritic cells enhances T cell activation and cytokine production. J Immunol. 2003; 170:1257-66. [PubMed: 12538684]

15. Latchman Y, Wood CR, Chernova T, et al. PD-L2 is a second ligand for PD-1 and inhibits T cell activation. Nat Immunol. 2001; 2:261-8. [PubMed: 11224527]

16. Brahmer J, Reckamp KL, Baas P, et al. Nivolumab versus docetaxel in advanced squamous-cell non-small-cell lung cancer. N Engl J Med. 2015; 373:123-35. [PubMed: 26028407]

17. Postow MA, Chesney J, Pavlick AC, et al. Nivolumab and ipilimumab versus ipilimumab in untreated melanoma. N Engl J Med. 2015; 372:2006-17. [PubMed: 25891304]

18. Larkin J, Chiarion-Sileni V, Gonzalez R, et al. Combined nivolumab and ipilimumab or monotherapy in untreated melanoma. N Engl J Med. 2015; 373:23-34. [PubMed: 26027431]

19. Robert C, Schachter J, Long GV, et al. Pembrolizumab versus ipilimumab in advanced melanoma. N Engl J Med. 2015; 372:2521-32. [PubMed: 25891173]

20. Wolchok JD, Kluger H, Callahan MK, et al. Nivolumab plus ipilimumab in advanced melanoma. N Engl J Med. 2013; 369:122-33. [PubMed: 23724867]

21. Hamid O, Robert C, Daud A, et al. Safety and tumor responses with lambrolizumab (Anti-PD-1) in melanoma. N Engl J Med. 2013; 369:134-44. [PubMed: 23724846]

22. Le DT, Uram JN, Wang H, et al. PD-1 blockade in tumors with mismatch-repair deficiency. N Engl J Med. 2015; 372:2509-20. [PubMed: 26028255]

23. Cancer Genome Atlas Research Network. Comprehensive molecular characterization of urothelial bladder carcinoma. Nature. 2014; 507:315-22. [PubMed: 24476821]

24. Damrauer JS, Hoadley KA, Chism DD, et al. Intrinsic subtypes of high-grade bladder cancer reflect the hallmarks of breast cancer biology. Proc Natl Acad Sci USA. 2014; 111:3110-5. [PubMed: 24520177]

25. Yadav M, Jhunjhunwala S, Phung QT, et al. Predicting immunogenic tumour mutations by combining mass spectrometry and exome sequencing. Nature. 2014; 515:572-6. [PubMed: 25428506]

26. Rizvi NA, Hellmann MD, Snyder A, et al. Cancer immunology. Mutational landscape determines sensitivity to PD-1 blockade in non-small cell lung cancer. Science. 2015; 348:124-8. [PubMed: 25765070]

27. Snyder A, Makarov V, Merghoub T, et al. Genetic basis for clinical response to CTLA-4 blockade in melanoma. N Engl J Med. 2014; 371:2189-99. [PubMed: 25409260]

28. Van Allen EM, Miao D, Schilling B, et al. Genomic correlates of response to CTLA-4 blockade in metastatic melanoma. Science. 2015; 350:207-11. [PubMed: 26359337]

29. Peng D, Kryczek I, Nagarsheth N, et al. Epigenetic silencing of TH1-type chemokines shapes tumour immunity and immunotherapy. Nature. 2015; 527:249-53. [PubMed: 26503055]

30. Herbst RS, Soria JC, Kowanetz M, et al. Predictive correlates of response to the anti-PD-L1 antibody MPDL3280A in cancer patients. Nature. 2014; 515:563-7. [PubMed: 25428504]

31. Chen DS, Irving BA, Hodi FS. Molecular pathways: next-generation immunotherapy--inhibiting programmed death-ligand 1 and programmed death-1. Clin Cancer Res. 2012; 18:6580-7. [PubMed: 23087408] 
32. Petrylak DP, Powles T, Bellmunt J, et al. A phase Ia study of MPDL3280A (anti-PDL1): Updated response and survival data in urothelial bladder cancer (UBC). J Clin Oncol. 2015; 33(suppl; abstr 4501)

33. Eisenhauer EA, Therasse P, Bogaerts J, et al. New response evaluation criteria in solid tumours: revised RECIST guideline (version 1.1). Eur J Cancer. 2009; 45:228-47. [PubMed: 19097774]

34. Nishino M, Tirumani SH, Ramaiya NH, Hodi FS. Cancer immunotherapy and immune-related response assessment: The role of radiologists in the new arena of cancer treatment. Eur J Radiol. 2015; 84:1259-68. [PubMed: 25937524]

35. Chiou VL, Burotto M. Pseudoprogression and immune-related response in solid tumors. J Clin Oncol. 2015; 33:3541-3. [PubMed: 26261262]

36. Powles T, Eder JP, Fine GD, et al. MPDL3280A (anti-PD-L1) treatment leads to clinical activity in metastatic bladder cancer. Nature. 2014; 515:558-62. [PubMed: 25428503]

37. Wu TD, Nacu S. Fast and SNP-tolerant detection of complex variants and splicing in short reads. Bioinformatics. 2010; 26:873-81. [PubMed: 20147302]

38. Law CW, Chen Y, Shi W, Smyth GK. voom: Precision weights unlock linear model analysis tools for RNA-seq read counts. Genome Biol. 2014; 15:R29. [PubMed: 24485249]

39. Ritchie ME, Phipson B, Wu D, et al. limma powers differential expression analyses for RNAsequencing and microarray studies. Nucleic Acids Res. 2015; 43:e47. [PubMed: 25605792]

40. Frampton GM, Fichtenholtz A, Otto GA, et al. Development and validation of a clinical cancer genomic profiling test based on massively parallel DNA sequencing. Nat Biotechnol. 2013; 31:1023-31. [PubMed: 24142049]

41. Kaplan EL, Meier P. Nonparametric estimation from incomplete observations. J Amer Statist Assn. 1958; 53:457-81.

42. Brookmeyer R, Crowley J. A confidence interval for the median survival time. Biometrics. 1982; 38:29-41.

43. Clopper C, Pearson ES. The use of confidence or fiducial limits illustrated in the case of the binomial. Biometrika. 1934; 26:404-13.

44. Sternberg CN, Yagoda A, Scher HI, et al. Preliminary results of M-VAC (methotrexate, vinblastine, doxorubicin and cisplatin) for transitional cell carcinoma of the urothelium. J Urol. 1985; 133:403-7. [PubMed: 4038749]

45. Choueiri TK, Ross RW, Jacobus S, et al. Double-blind, randomized trial of docetaxel plus vandetanib versus docetaxel plus placebo in platinum-pretreated metastatic urothelial cancer. $\mathrm{J}$ Clin Oncol. 2012; 30:507-12. [PubMed: 22184381]

46. Bambury RM, Benjamin DJ, Chaim JL, et al. The safety and efficacy of single-agent pemetrexed in platinum-resistant advanced urothelial carcinoma: a large single-institution experience. Oncologist. 2015; 20:508-15. [PubMed: 25845990]

47. Agarwal N, Bellmunt J, Maughan BL, et al. Six-month progression-free survival as the primary endpoint to evaluate the activity of new agents as second-line therapy for advanced urothelial carcinoma. Clin Genitourin Cancer. 2014; 12:130-7. [PubMed: 24220220]

48. Boorjian SA, Sheinin Y, Crispen PL, et al. T-cell coregulatory molecule expression in urothelial cell carcinoma: clinicopathologic correlations and association with survival. Clin Cancer Res. 2008; 14:4800-8. [PubMed: 18676751]

49. Bellmunt J, Mullane SA, Werner L, et al. Association of PD-L1 expression on tumor-infiltrating mononuclear cells and overall survival in patients with urothelial carcinoma. Ann Oncol. 2015; 26:812-7. [PubMed: 25600565] 


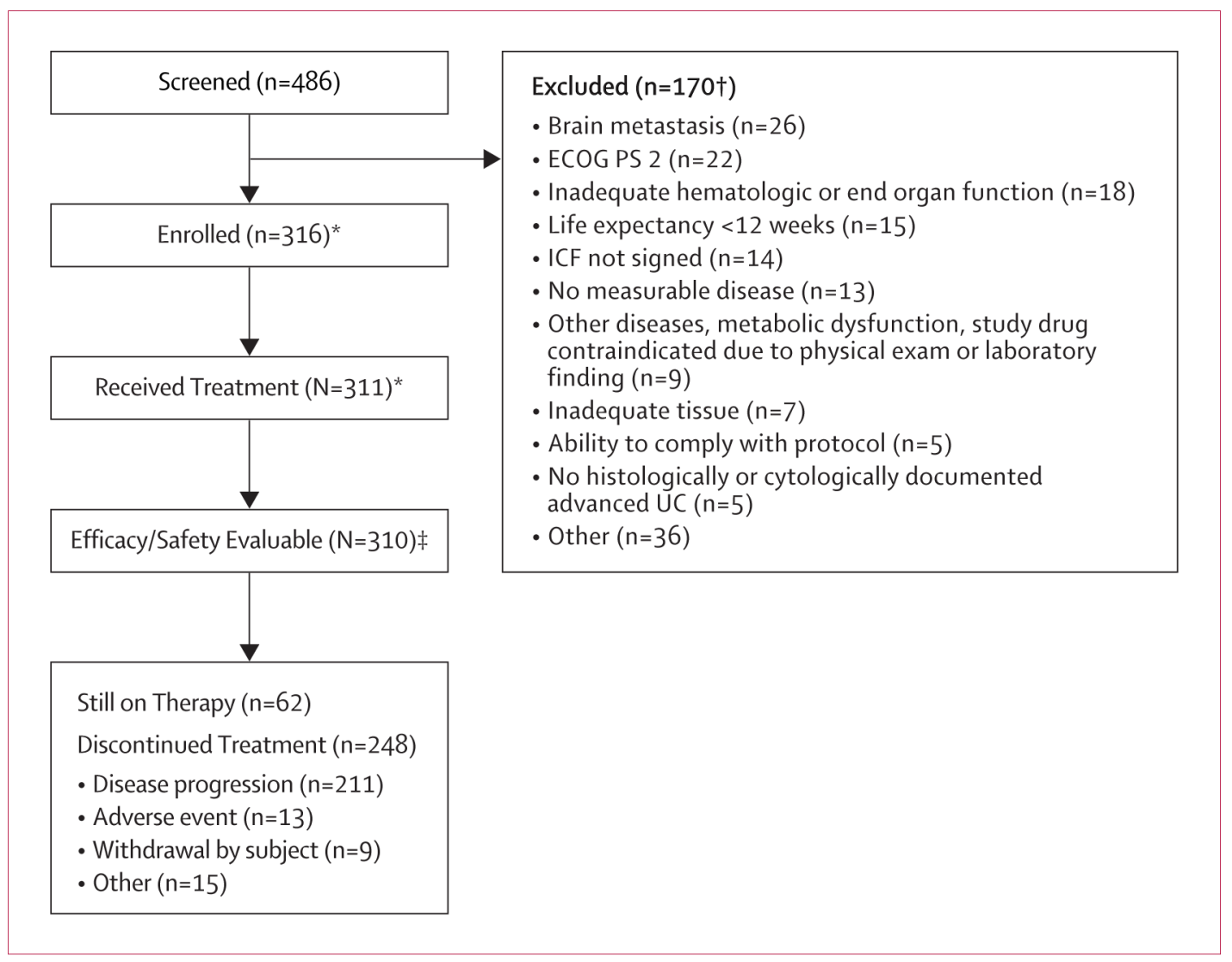

Figure 1. Trial profile for Cohort 2

*Based on May 5, 2015 data cut. Two Cohort 2 patients and one Cohort 1 patient were reassigned to the alternate cohort based on eligibility reassessments between the May 5 and September 14, 2015 data cuts (enrolled and treated n's based on September data cut are 315 and 310 , respectively).

$\ddagger$ Excludes 1 patient with unknown site.

$\dagger$ Includes rescreened patients.

ECOG PS, Eastern Cooperative Oncology Group performance status; ICF, informed consent form; UC, urothelial carcinoma. 


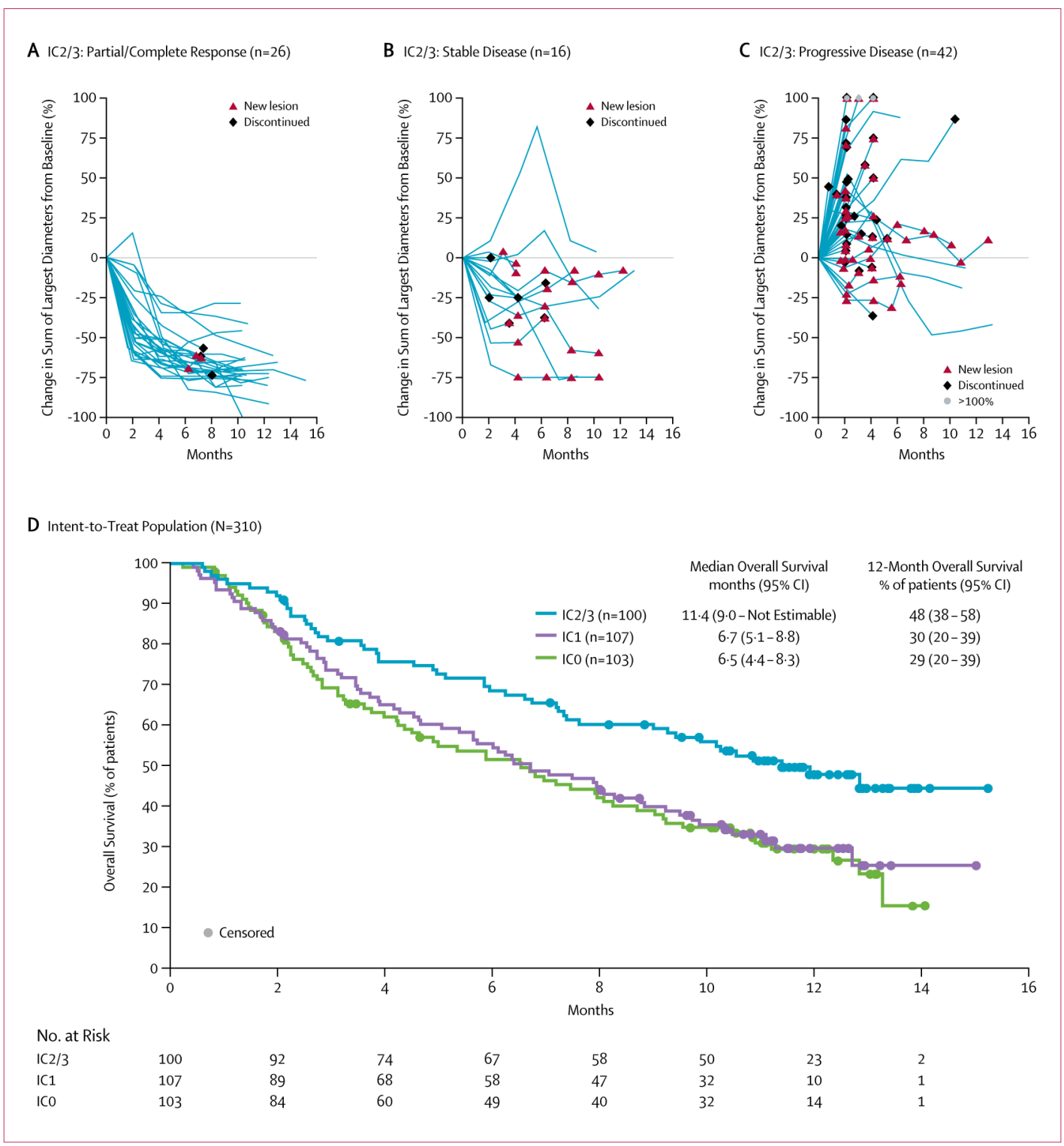

Figure 2. Change in sum of longest diameters over time by best response in the PD-L1 IC2/3 group and comparison of overall survival among PD-L1 IC groups

Percent change in the sum of longest diameters (SLD) by independent review assessed RECIST v1.1 in the IC2/3 group by (A) responders; (B) stable disease; (C) progressive disease. Patients without a measurable baseline tumor assessment or without post-baseline tumor measurements were not included. (D) Kaplan-Meier overall survival curves for the IC0, IC1, and IC2/3 groups.

IC, immune cell; CI, confidence interval. 


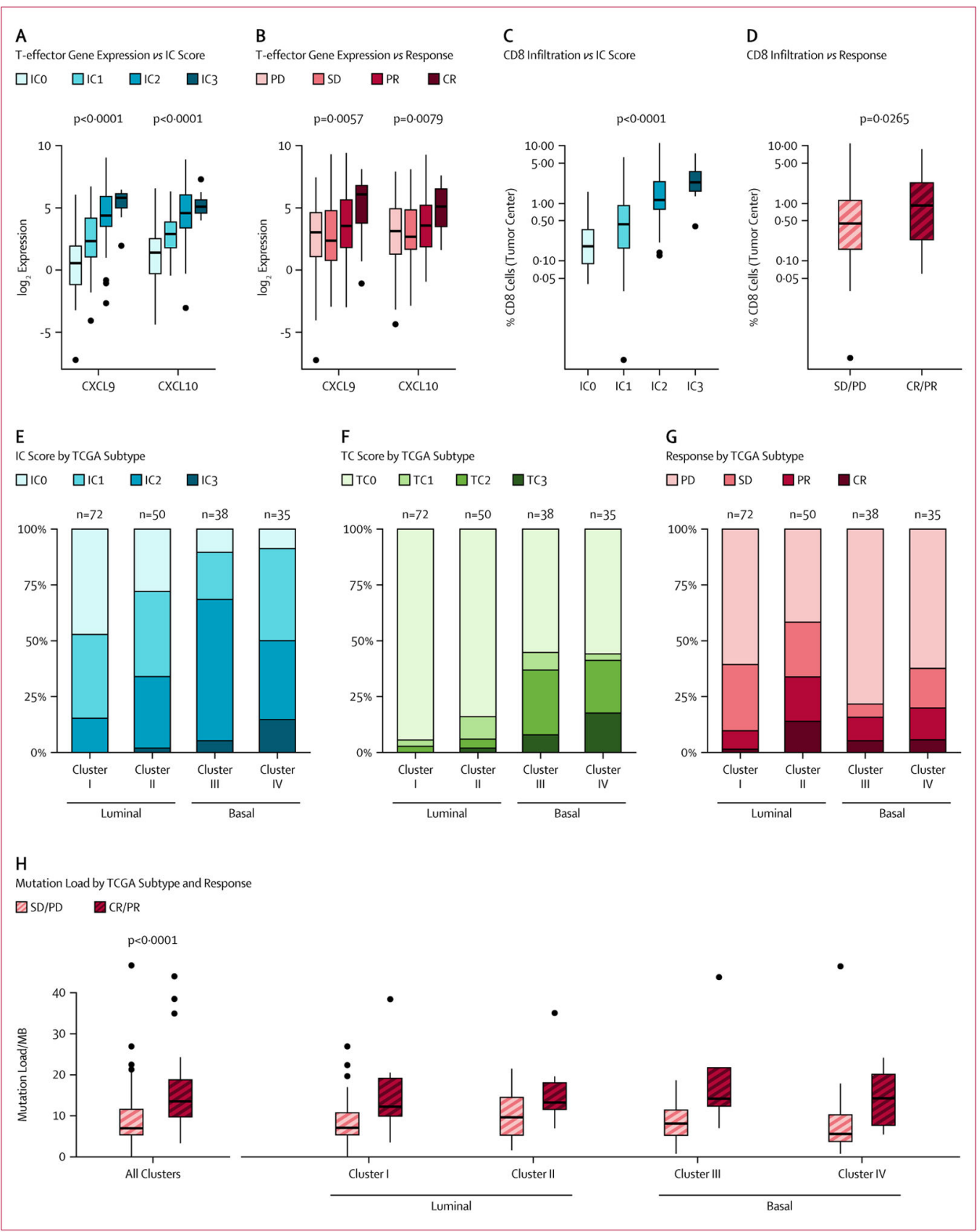

Figure 3. Association of response and PD-L1 IHC status with gene expression profiling and mutation load

(A) Association of PD-L1 IHC IC with gene expression for CXCL9 and CXCL10, two representatives of a CD8 $\mathrm{T}$ effector gene set. For each gene, expression increased linearly with IC score $(\mathrm{p}<0.0001$ and $\mathrm{p}<0.0001$, respectively). The other genes in an eight-gene CD8 $\mathrm{T}$ effector gene set behaved similarly (figure 6A, appendix). (B) CXCL9 and CXCL10 were significantly associated with response $(\mathrm{p}=0.0057$ and $\mathrm{p}=0.0079$, respectively). The other genes in the set did not achieve statistical significance individually, likely due to lower RNAseq read counts, but they exhibited behavior qualitatively similar to that of CXCL9 and 
CXCL10 (figure 6B, appendix). (C) Tumor CD8+ T cell infiltration in the tumor center was significantly associated with PD-L1 IC ( $\mathrm{p}<0 \cdot 0001)$. (D) Response was significantly associated with increased CD8+ IHC staining in the tumor center $(p=0.0265)$. (E) IC score distribution by TCGA subtype. IC2/3 was significantly more common in the basal subtypes (III and IV, p $<0 \cdot 0001$ ), though IC2 was present in all subtypes. (F) TC score distributions by TCGA subtype. TC2/3 was almost exclusively seen in basal subtypes ( $<<0 \cdot 0001)$. (G) Objective response rate by TCGA subtype. Response was significantly associated with TCGA subtype: $10 \%$ for subtype I, $34 \%$ for II, $16 \%$ for III, and $20 \%$ for IV ( $\mathrm{p}=0.0102)$. (H) Estimated mutation load per megabase $(\mathrm{Mb})$ vs patient response, overall $(\mathrm{n}=150)$ and also disaggregated by TCGA subtype. Mutation load was strongly associated with response $(\mathrm{p}<0 \cdot 0001)$, but to a similar degree in all TCGA subtypes.

IC, immune cell; TC, tumor cell; CR, complete response; PR, partial response; SD, stable disease; PD, progressive disease. 
Table 1

Baseline patient characteristics and prior therapy

\begin{tabular}{|c|c|c|c|}
\hline Characteristic & $\begin{array}{l}\text { IC2/3 } \\
\mathbf{n}=100\end{array}$ & $\underset{\mathbf{n}=\mathbf{2 0 7}}{\mathrm{IC} 1 / 2 / 3}$ & $\begin{array}{c}\text { All Patients } \\
\mathbf{N}=\mathbf{3 1 0}\end{array}$ \\
\hline Age, Median, years (range) & $66(41-84)$ & $67(32-91)$ & $66(32-91)$ \\
\hline Sex, male, n $(\%)$ & $78(78)$ & $160(77)$ & $241(78)$ \\
\hline Race, Caucasian, n (\%) & $87(87)$ & $184(89)$ & $282(91)$ \\
\hline \multicolumn{4}{|l|}{ Site of primary tumor, $\mathrm{n}(\%)$} \\
\hline Bladder & $79(79)$ & $159(77)$ & $230(74)$ \\
\hline Renal pelvis & $11(11)$ & $27(13)$ & $42(14)$ \\
\hline Ureter & $5(5)$ & $12(6)$ & $23(7)$ \\
\hline Urethra & $3(3)$ & $5(2)$ & $5(2)$ \\
\hline Other & $2(2)$ & $4(2)$ & $10(3)$ \\
\hline Baseline creatinine clearance, $<60 \mathrm{~mL} / \mathrm{min}, \mathrm{n}(\%)$ & $40(40)$ & $69(33)$ & $110(36)$ \\
\hline \multicolumn{4}{|l|}{ ECOG PS, n (\%) } \\
\hline 0 & $42(42)$ & $83(40)$ & $117(38)$ \\
\hline 1 & $58(58)$ & $124(60)$ & $193(62)$ \\
\hline Hemoglobin, <10 g/dL, n (\%) & $24(24)$ & $50(24)$ & $69(22)$ \\
\hline \multicolumn{4}{|l|}{ Tobacco use, n (\%) } \\
\hline Current & $6(6)$ & $19(9)$ & $35(11)$ \\
\hline Never & $34(34)$ & $72(35)$ & $107(35)$ \\
\hline Previous & $60(60)$ & $116(56)$ & $168(54)$ \\
\hline \multicolumn{4}{|l|}{ Bellmunt risk factors, number, $\mathrm{n}(\%)$} \\
\hline 0 & $31(31)$ & $61(30)$ & $83(27)$ \\
\hline 1 & $35(35)$ & $72(35)$ & $117(38)$ \\
\hline 2 & $28(28)$ & $59(29)$ & $89(29)$ \\
\hline 3 & $6(6)$ & $15(7)$ & $21(7)$ \\
\hline \multicolumn{4}{|l|}{ Metastatic sites at baseline, $\mathrm{n}(\%)$} \\
\hline Visceral $^{a}$ & $66(66)$ & $152(73)$ & $243(78)$ \\
\hline Liver & $27(27)$ & $61(30)$ & $96(31)$ \\
\hline Lymph node only & $24(24)$ & $39(19)$ & $43(14)$ \\
\hline Prior cystectomy, yes, n (\%) & $44(44)$ & $83(40)$ & $115(37)$ \\
\hline Time from prior chemotherapy $₫$ months, $\mathrm{n}(\%)$ & $43(43)$ & $87(42)$ & $121(39)$ \\
\hline \multicolumn{4}{|l|}{ Prior therapy with platinum-based regimen, n (\%) } \\
\hline Cisplatin-based & $83(83)$ & $161(78)$ & $227(73)$ \\
\hline Carboplatin-based & $17(17)$ & $43(21)$ & $80(26)$ \\
\hline Other platinum combination & 0 & $3(1)$ & $3(1)$ \\
\hline Prior neoadjuvant or adjuvant chemotherapy, with first progression $\leq 2$ months, $\mathrm{n}$ (\%) & $24(24)$ & $42(20)$ & $57(18)$ \\
\hline \multicolumn{4}{|l|}{ Number of prior systemic regimens in the metastatic setting, $\%$} \\
\hline 0 & $24(24)$ & $42(20)$ & $59(19)$ \\
\hline
\end{tabular}




\begin{tabular}{|l|c|c|c|}
\hline Characteristic & $\begin{array}{c}\text { IC2/3 } \\
\mathbf{n}=\mathbf{1 0 0}\end{array}$ & $\begin{array}{c}\text { IC1/2/3 } \\
\mathbf{n = 2 0 7}\end{array}$ & $\begin{array}{c}\text { All Patients } \\
\mathbf{N = 3 1 0}\end{array}$ \\
\hline 1 & $36(36)$ & $83(40)$ & $124(40)$ \\
\hline 2 & $19(19)$ & $41(20)$ & $64(21)$ \\
\hline 3 & $11(11)$ & $24(12)$ & $39(13)$ \\
\hline$\geq 4$ & $10(10)$ & $17(8)$ & $24(8)$ \\
\hline Intravesical bacillus Calmette-Guérin administered, n (\%) & $15(15)$ & $46(22)$ & $73(24)$ \\
\hline
\end{tabular}

IC, immune cell; ECOG PS, Eastern Cooperative Oncology Group performance status.

${ }^{a}$ Visceral metastasis defined as liver, lung, bone, any non-lymph node or soft tissue metastasis.

Data cutoff: September 14, 2015. 
Table 3

Treatment-related adverse events in the 310 patients receiving atezolizumab

\begin{tabular}{|c|c|c|}
\hline Event & $\begin{array}{c}\text { All Grade } \\
\text { n (\%) }\end{array}$ & $\begin{array}{c}\text { Grade 3-4 } \\
\text { n }(\%)\end{array}$ \\
\hline Any AE & $215(69)$ & $50(16)$ \\
\hline Fatigue & $93(30)$ & $5(2)$ \\
\hline Nausea & $42(14)$ & $0(0)$ \\
\hline Decreased Appetite & $36(12)$ & $2(1)$ \\
\hline Pruritis & $31(10)$ & $1(<1)$ \\
\hline Pyrexia & $28(9)$ & $1(<1)$ \\
\hline Diarrhea & $24(8)$ & $1(<1)$ \\
\hline Rash & $23(7)$ & $1(<1)$ \\
\hline Arthralgia & $21(7)$ & $2(1)$ \\
\hline Vomiting & $18(6)$ & $1(<1)$ \\
\hline Dyspnea & $10(3)$ & $2(1)$ \\
\hline Anemia & $9(3)$ & $3(1)$ \\
\hline Aspartate aminotransferase increased & $10(3)$ & $2(1)$ \\
\hline Pneumonitis & $7(2)$ & $2(1)$ \\
\hline Hypotension & $5(2)$ & $2(1)$ \\
\hline Hypertension & $3(1)$ & $3(1)$ \\
\hline Colitis & $3(1)$ & $2(1)$ \\
\hline
\end{tabular}

Data cutoff: September 14, 2015. 